\title{
PARTICLE PHYSICS
}

-at the start of the NEW MILLENNIUM 
This page is intentionally left blank 


\title{
INTERREGIONAL CENTRE
FOR ADVANCED STUDIES
}

\author{
Proceedings of the Ninth Lomonosov \\ Conference on Elementary Particle Physics
}

\section{PARTICLE PHYSICS \\ at the start of the \\ NEW MILLENNIUM}

20-26 September 1999, Moscow

\author{
Editor \\ Alexander I Studenikin \\ Department of Theoretical Physics \\ Moscow State University, Russia
}




\section{Published by}

World Scientific Publishing Co. Pte. Ltd.

P O Box 128, Farrer Road, Singapore 912805

USA office: Suite 1B, 1060 Main Street, River Edge, NJ 07661

UK office: 57 Shelton Street, Covent Garden, London WC2H 9HE

\section{British Library Cataloguing-in-Publication Data}

A catalogue record for this book is available from the British Library.

\section{PARTICLE PHYSICS AT THE START OF THE NEW MILLENNIUM}

Copyright $\odot 2001$ by World Scientific Publishing Co. Pte. Ltd.

All rights reserved. This book, or parts thereof, may not be reproduced in any form or by any means, electronic or mechanical, including photocopying, recording or any information storage and retrieval system now known or to be invented, without written permission from the Publisher.

For photocopying of material in this volume, please pay a copying fee through the Copyright Clearance Center, Inc., 222 Rosewood Drive, Danvers, MA 01923, USA. In this case permission to photocopy is not required from the publisher.

ISBN 981-02-4644-7

Printed in Singapore by World Scientific Printers 


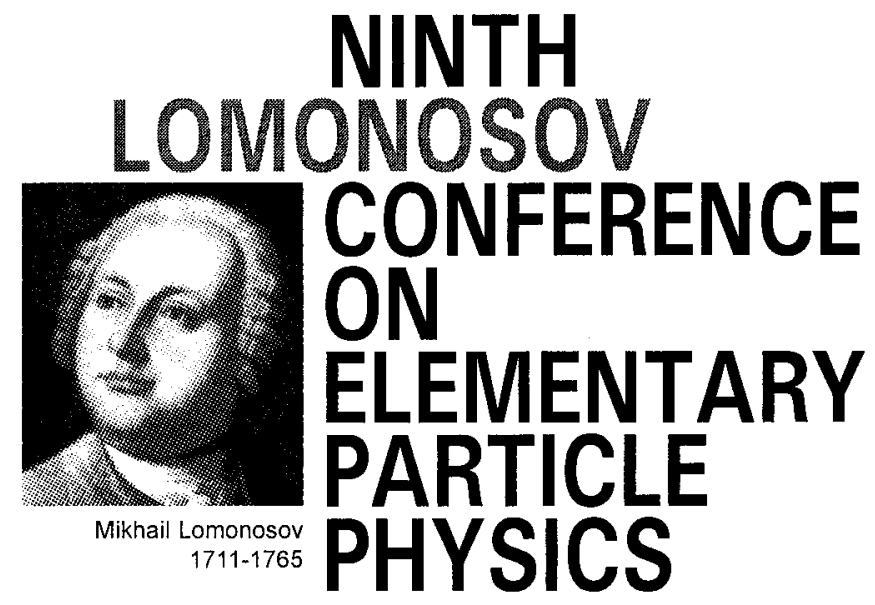

Sponsors

Russian Foundation for Basic Research

Interregional Centre for Advanced Studies

Ministry of Science and Technology of Russia

\section{Supporting Institutions}

Faculty of Physics and Institute of Theoretical Microphysics of Moscow State University

Joint Institute for Nuclear Research (Dubna)

Instituto Superior Tecnico - CENTRA (Lisbon)

Institute of Theoretical and Experimental Physics (Moscow)

Institute for Nuclear Research (Moscow)

Institute for High Energy Physics (Protvino) 


\section{Programme Committee}

V.Bagrov (Tomsk State Univ.), V.Belokurov (MSU), V.Braginsky (MSU),

G.Diambrini-Palazzi (Univ. of Rome),

J.Dias de Deus (IST/CENTRA, Lisbon),

D.Ebert (Humboldt Univ., Berlin), R.Faustov (NSK, RAN \& ICAS, Moscow),

D.Galtsov (MSU), V.Kadyshevsky (JINR, Dubna), A.Logunov (IHEP, Protvino), V.Matveev (INR, Moscow),

V.Melnikov (Russian Grav. Soc., Moscow),

V.Mostepanenko (PTI, St.-Petersburg),

A.Mourao (IST/CENTRA, Lisbon),

A.Nikishov (Lebedev Physical Inst., Moscow),

L.Okun (ITEP, Moscow), V.Ritus (Lebedev Physical Inst., Moscow),

V.Rubakov (INR, Moscow), V.Savrin (MSU), F.Selleri (Univ. of Bari),

D.Shirkov (JINR, Dubna), Yu.Simonov (ITEP, Moscow),

A.Slavnov (MSU \& Steklov Math. Inst., Moscow),

A.Smirnov (INR, Moscow \& ICTP, Trieste),

P.Spillantini (INFN, Florence), A.Studenikin (MSU \& ICAS, Moscow),

V.Trukhin (MSU), V.Zhukovsky (MSU)

\section{Organizing Committee}

G.Diambrini-Palazzi (Univ. of Rome),

J.Dias de Deus (IST/CENTRA, Lisbon),

A.Egorov (MSU \& ICAS, Moscow) - Secretary,

R.Faustov (Russian Academy of Sciences \& ICAS, Moscow),

V.Galkin (Russian Academy of Science \& CAS, Moscow),

A.Kataev (INR, Moscow), O.Khrustalev (MSU), P.Kashkarov (MSU),

V.Melnikov (Russian Grav. Soc., Moscow), V.Mikhailin (MSU),

A.Mourao (IST/CENTRA, Lisbon), N.Nikitina (MSU),

P.Nowosad (Univ. of Sao Paulo), Yu.Popov (MSU),

V.Rubakov (INR, Moscow), P.Silaev (ITM, Moscow State University)

A.Sisakian (JINR, Dubna),

A.Slavnov (MSU \& Steklov Math. Inst., Moscow),

A.Studenikin (MSU \& ICAS, Moscow) - Chairman,

V.Trukhin (MSU), Yu.Vladimirov (MSU) 


\section{FOREWORD}

The $9^{\text {th }}$ Lomonosov Conference on Elementary Particle Physics was held at the Moscow State University (Moscow, Russia) on September 20-26, 1999.

The conference was organized by the Faculty of Physics of the Moscow State University and the Interregional Centre for Advanced Studies in co-operation with the Institute of Theoretical Microphysics of the Moscow State University and supported by the Joint Institute for Nuclear Research (Dubna), the Instituto Superior Tecnico-CENTRA (Lisbon), the Institute of Theoretical and Experimental Physics (Moscow), the Institute for High Energy Physics (Protvino) and the Institute for Nuclear Research (Moscow). The Ministry of Science and Technology of Russia and the Russian Foundation for Basic Research also sponsored the conference.

It was eighteen years ago when the first of the series of conferences (from 1993 called the "Lomonosov Conferences"), was held at the Department of Theoretical Physics of the Moscow State University (June 1983, Moscow). The second conference was held in Kishinev, Republic of Moldavia, USSR (May 1985).

After the four years break this series was resumed on a new conceptual basis for the conference programme focus. During the preparation of the third conference (that was held in Maykop, Russia, 1989) a desire to broaden the programme to include more general issues in particle physics became apparent. At subsequent meetings of this series (Minsk, Republic of Byelorussia, USSR, 1990; Yaroslavl, Russia, 1992) a wide variety of interesting things both in theory and experiment of particle physics, field theory, gravitation and astrophysics were included into the programmes. During the conference of 1992 in Yaroslavl it was proposed by myself and approved by numerous participants that these irregularly held meetings should be transformed into regular events under the title "Lomonosov Conferences on Elementary Particle Physics". It was also decided to enlarge the number of institutions that would take part in preparation of future conferences.

Mikhail Lomonosov (1711-1765), a brilliant Russian encyclopaedias of the era of the Russian Empress Catherine the 2nd, was world renowned for his distinguished contributions in the fields of science and art. He also helped establish the high school educational system in Russia. The Moscow State University was founded in 1755 based on his plan and initiative, and the University now bears the name of Lomonosov.

The $6^{\text {th }}$ Lomonosov Conference on Elementary Particle Physics "Cosmomicrophysics and Gauge Fields" was held at the Moscow State University (1993). The publication of the volume containing articles written on the basis of presentations at the $5^{\text {th }}$ and $6^{\text {th }}$ Lomonosov Conferences was supported by the Accademia Nazionale dei Lincei (Italy). 
viii

The $7^{\text {th }}$ and $8^{\text {th }}$ Lomonosov Conference (1995 and 1997) were also held in Moscow and the proceedings of these meetings entitled "Problems of Fundamental Physics" and "Elementary Particle Physics" were published by the Interregional Centre for Advanced Studies.

The physics programme of the $9^{\text {th }}$ Lomonosov Conference included 27 review talks and 45 session reports on various topics in particle physics, as fundamental symmetries, electroweak theory, tests of standard model and beyond, heavy quark physics, non-perturbative QCD, neutrino physics, astroparticle physics, quantum gravity effects.

On behalf of the Organizing Committee I should like to warmly thank the session chairpersons, the speakers and all of the participants of the 9th Lomonosov Conference.

We are grateful to the Dean of the Faculty of Physics of the Moscow State University, Vladimir Trukhin, the Directors of the Joint Institute for Nuclear Research, Vladimir Kadyshevsky, the Director of the Institute of Theoretical and Experimental Physics, Mikhail Danilov, and the Dean of the Faculty of Physics of the Instituto Superior Tecnico-CENTRA, Jorge Dias de Deus, for the support in organizing the conference.

I should like to thank Lev Okun and Andrey Kataev for their help in planning of the scientific programme of the meeting. Special thanks are due to Ana Mourao, Andrei Kounine and Boleslaw Pietrzyk for their valuable help in inviting speakers for the topical sessions of the conference.

Furthermore, I am very pleased to mention Andrey Egorov, the Scientific Secretary of the conference, and Alexey Illarionov for their very efficient work in preparing and running the meeting.

These Proceedings were prepared for publication and sponsored by the Interregional Centre for Advanced Studies with support by the Ministry of Science and Technology of Russia and the Russian Foundation for Basic Research. 


\section{CONTENTS}

Sponsors and Committees

Foreword

vii

On the Fundamental Symmetries in Particle Physics

E. Shabalin

Chiral Symmetry in Lattice QCD
A. Slavnov

NA48 Rare Decay Results

B. Vallage

Two Photon Physics at LEP

G. Passaleva

Adler Function from $\mathrm{R}^{\mathrm{e}+\mathrm{e}}(\mathrm{s})$ Measurements: Experiments vs QCD Theory

\section{A. Kataev}

Perturbation Theory with Convergent Series for Large Values of

Coupling Constant: A Calculation of $\beta$-Function in $\varphi^{4}$ Model

V. Belokurov, E. Shavgulidze, Yu. Solov'yov and I. Yudin

Two-Dimensional QCD and Instanton Contribution

A. Bassetto, F. Vian and L. Griguolo

Color Reconnection and Bose-Einstein Correlations at LEP2

Th. Ziegler

Analytic Approach to QCD and the Interquark Potential

A. Nesterenko

A NLO QCD Analysis of the Spin Structure Function $g_{1}$ and Higher Twist Corrections

E. Leader, A. Sidorov and D. Stamenov 
Leptonic Decays of Ds

J. Putz

Heavy Quark Asymmetries

A. Tricomi

Experimental Signature of a Fermiophobic Higgs Boson

L. Brïecher and R. Santos

S-Channel Higgs Physics beyond the Standard Model

G. Boyarkina

Quantum Field of Nonstationary Polaron

O. Khrustalev, M. Tchitchikina and E. Spirina

Dirac's Equation, SU(2) Model and Octanions

S. Kopylov

Irreducible Darboux Transformations

V. Bagrov and B. Samsonov

The AMS Experiment: First Results and Physics Prospects

J. $P$. Vialle

Possible Observational Evidences of Non-Compact

(Non-Baryonic) Microlenses ("Neutralino Stars")

A. Zakharov

Neutrino Conversions in Active Galactic Nuclei

A. Husain

Lepton Production by Neutrinos in an External Electromagnetic Field

A. Borisov and N. Zamorin

Propagation of Axions in a Strongly Magnetized Medium

A. Borisov and P. Sizin

Mixing and CP Violation with Quasidegenerate Majorana Neutrinos 
Solar Neutrino Oscillations in Extensions of the Standard Model

O. Boyarkin

Covariant Treatment of Neutrino Spin (Flavour) Conversion in

Matter under the Influence of Electromagnetic Fields

M. Dvornikov, A. Egorov, A. Lobanov and A. Studenikin

Pulsar Velocity Puzzle and Nonstandard Neutrino Oscillations

R. Horvat

Kinematic Projecting of Pulsar Profiles

V. Bordovitsyn, V. Epp and V. Bulenok

Neutral Particle Radiation in Electromagnetic Field

A. Lobanov and O. Pavlova

Radiation of Relativistic Particles in a Quasi-Homogeneous

Magnetic Field

V. Epp and T. Mitrofanova

Spin-Orbital Motion and Thomas Precession in the Classical and Quantum Theories

V. Bordovitsyn and A. Myagkii

Late Gravitational Collapse, Quantum Miniholes and the Birth of a New Universe

M. Fil'chenkov

On Adelic Strings

B. Dragovich

Collider Searches for TeV Scale Quantum Gravity with Compact Extra Dimensions

P. Azzurri

Dynamics of Event Horizon Creation

A. Shatsky

Conference Programme 